\title{
Long-term cosmesis following a novel schedule of accelerated partial breast radiation in selected early stage breast cancer: result of a prospective clinical trial
}

\author{
Mutlay Sayan, MD¹, Daphne Hard, BS¹, Karen Wilson, MEd, CCRP², Carl Nelson, MD¹, Havaleh Gagne, MD', \\ Deborah Rubin, MD', Ruth Heimann, MD, PhD' \\ 'Department of Radiation Oncology, University of Vermont Medical Center, Burlington, VT, USA; \\ ${ }^{2}$ University of Vermont Cancer Center, Burlington, VT, USA
}

Purpose: There is controversy regarding the cosmetic outcome after accelerated partial breast radiation (APBR). We report the cosmetic outcome from a single-arm prospective clinical trial of APBR delivered using intensity-modulated radiation therapy (IMRT) in elderly patients with stage I breast cancer (BC), using a novel fractionation schedule.

Materials and Methods: Forty-two patients aged $\geq 65$, with Stage I BC who underwent breast-conserving surgery were enrolled in a phase I/II study evaluating a 2-week course of APBR. Thirty eligible patients received 40 Gy in 4 Gy daily fractions. Cosmetic outcome was assessed subjectively by physician/patient and objectively by using a computer program (BCCT.core) before APBR, during, and after completion of the treatment.

Results: The median age was 72 years, the median tumor size was $0.8 \mathrm{~cm}$, and the median follow-up was 50.5 months. The 5-year locoregional control in this cohort was $97 \%$ and overall survival $87 \%$. At the last follow-up, patients and physicians rated cosmesis as 'excellent' or 'good' in 100\% and $91 \%$, respectively. The BCCT.core program scored the cosmesis as 'excellent' or 'good' in $87 \%$ of the patients at baseline and $81 \%$ at the last follow-up. The median $V_{50}(20 \mathrm{~Gy})$ of the whole breast volume (WBV) was 37.2\%, with the median WBV V 100 (40 Gy) of 10.9\%.

Conclusion: An excellent rate of tumor control was observed in this prospective trial. By using multiple assessment techniques, we are showing acceptable cosmesis, supporting the use of IMRT planned APBR with daily schedule in elderly patients with early stage BC.

Keywords: Breast neoplasms, Dose hypofractionation, Intensity modulated therapy

\section{Introduction}

Breast-conserving surgery (BCS) and whole breast radiation (WBR) for women with early-stage breast cancer has become a standard of care as it shown to be equivalent to mastectomy for local control and survival. However, adjuvant WBR requires up to 6 weeks of daily radiation treatment, leading to underutilization of WBR by elderly patients and those who reside long distance from treatment facilities. Recently, accelerated partial breast radiation (APBR) has been proposed as an alternative to WBR for women with early-stage breast cancer as it reduces treatment time. While several investigators

Received 03 April 2017, Revised 06 July 2017, Accepted 10 July 2017.

Correspondence: Ruth Heimann, MD, PhD, Department of Radiation Oncology, University of Vermont Medical Center, 111 Colchester Avenue, Burlington, VT 05401, USA. Tel: +1-802-847-3506, Fax: +1-802-847-7413, E-mail: Ruth.Heimann@uvmhealth.org

(c) This is an Open Access article distributed under the terms of the Creative Commons Attribution Non-Commercial License (http://creativecommons.org/ licenses/by-nc/4.0/) which permits unrestricted non-commercial use, distribution, and reproduction in any medium, provided the original work is properly cited.

www.e-roj.org 
reported excellent local control among appropriately selected patients, the concern remains about long-term cosmetic outcome.

Several techniques for APBR have been reported including multicatheter brachytherapy [1], MammoSite brachytherapy [2] and external beam radiation therapy (EBRT) with threedimensional conformal radiation therapy (3D-CRT) or intensity-modulated radiation therapy (IMRT) [3,4]. IMRT improves conformal dose coverage [5] and provides better normal tissue sparing compared to 3D-CRT $[6,7]$. It allows control of the dose in the untreated part of the breast which theoretically should improve the cosmesis. There are only a limited number of studies using IMRT, and results regarding the cosmesis using APBR are conflicting. Most of the reported schedules of APBR used twice a day schedule which may have impacted on the cosmesis as the normal breast tissue may not have adequate time to recover. Twice a day schedules are also difficult on patients particularly elderly and those travel long distance. Therefore we designed our trial of APBR using daily treatments.

Subjective evaluation by the physicians and/or patient self-assessment is commonly used to report cosmesis [8]. Subjective evaluation by physicians has low reproducibility and self-assessment is more dependent on psycho-social adjustment than of the real cosmesis [9-11]. In order to overcome this issue, a computer program, BCCT.core, was developed to provide objective evaluation based on breast symmetry, skin color changes, and surgical scar appearance by using photographs of the patients taken during the followups [12]. BCCT.core has been shown to assess the cosmesis accurately in multiple studies by providing consistent and reproducible results [13].

We previously reported excellent tumor control with acceptable toxicity [14]. We are reporting here cosmetic outcome and the dosimetric parameters. To our knowledge, this study is the first comprehensive investigation of cosmesis by using both subjective and objective evaluation with dosimetric assessment in patients treated with APBR.

\section{Methods and Materials}

\section{Study population}

Forty-two patients with stage I breast cancer were enrolled in this phase I/II study at University of Vermont Cancer Center between June 2006 and May 2013. The Institutional Review Board approved the study (IRB No. VCC-0601). The study was conducted in accordance with the principles of the Declaration of Helsinki and all patients provided written informed consent.

Eligibility criteria included: (1) $\geq 65$ years old female patient with AJCC stage I breast cancer, post-partial mastectomy and axillary node dissection or sentinel lymph node biopsy; (2) visible lumpectomy cavity on planning computed tomography

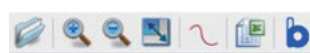

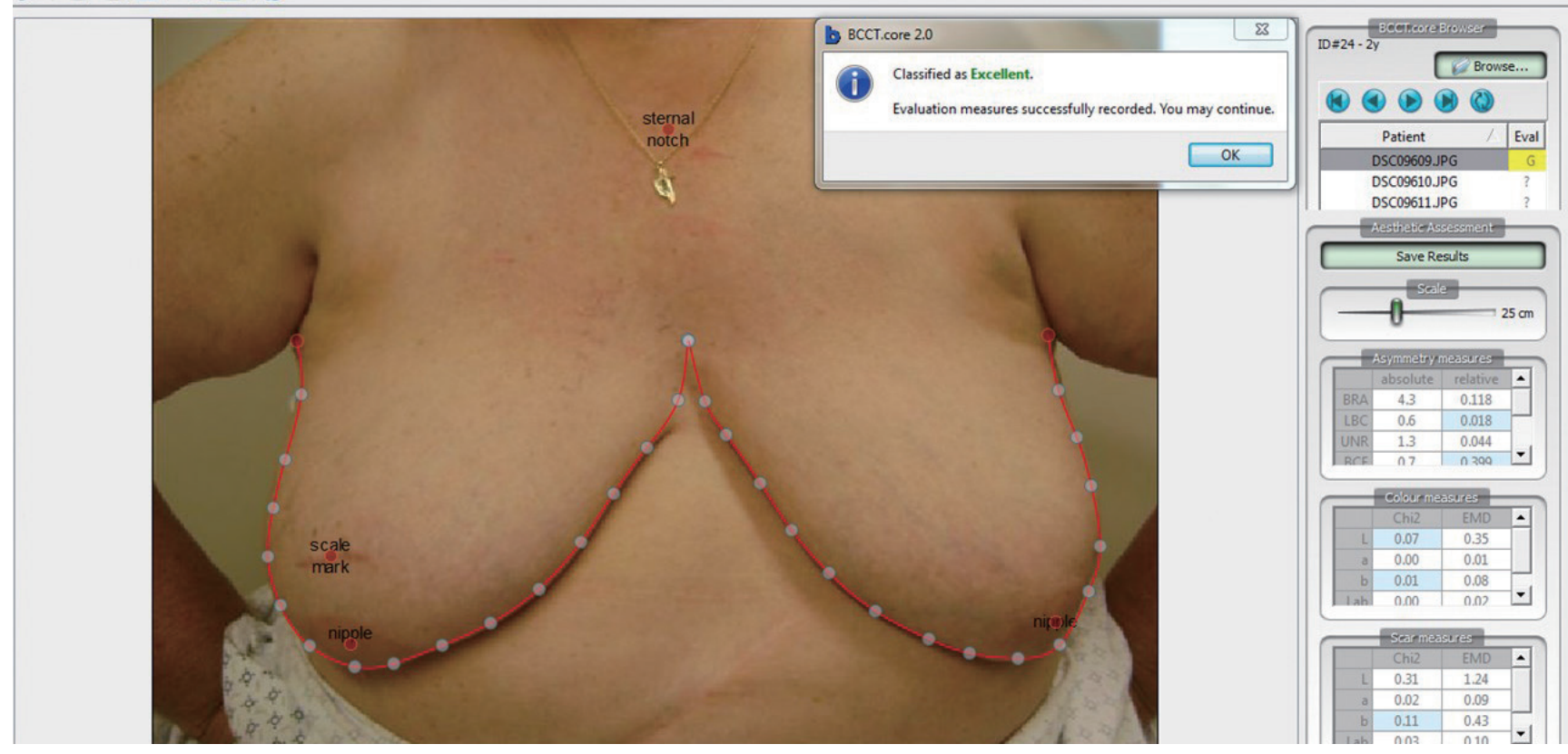

Fig. 1. The BCCT.core screen layout. 
(CT); and (3) ability to begin radiation treatment 3-8 week post-surgery. If indicated, hormonal and chemotherapy was allowed. Patients with lymphovascular invasion (LVI) or positive tumor margins were not eligible. Only patients with photographs at baseline and from follow-up visits at $\geq 2$ years were included in this analysis.

\section{Simulation and treatment technique}

Patients were simulated lying supine with both arms raised above the head on a breast board with Vacloc immobilization. Based on the planning CT scan, the lumpectomy cavity, both breasts, both lungs, and the heart were contoured. The clinical target volume (CTV) was defined as the excision cavity +1.0 $\mathrm{cm}$ (5 $\mathrm{mm}$ from skin surface and from pectoralis muscle). The planning target volume (PTV) was a $0.5 \mathrm{~cm}$ expansion around the CTV. The final PTV was modified to exclude the first $5 \mathrm{~mm}$ of tissue from the skin surface and the portion extending into the lung.

IMRT was used for treatment planning using Philips Pinnacle planning system (Philips Healthcare, Best, The Netherlands). Planning guidelines were to cover the PTV with at least 95\% isodose line. Ten fractions of 4 Gy were delivered once daily 5 days per week for a total dose of $40 \mathrm{~Gy}$.

\section{Dosimetric assessment}

The whole breast volume (WBV) was individually outlined to include the glandular tangent fields. The volume of the whole breast receiving 5\%,20\%,50\%,80\%, and 100\% of the prescription dose $\left(V_{51}, V_{20}, V_{50}, V_{80}\right.$, and $\left.V_{100}\right)$ was recorded. Outside of the target breast volume (WBV-PTV) receiving the 5\%-100\% of the prescription dose $\left(V_{5}-V_{100}\right)$ was determined. Maximum dose (Dmax), PTV, and PTV to WBV ratio was also assessed.

\section{Patient follow-up}

Patients were seen weekly while on treatment and for an additional 4 weeks by the treating radiation oncologist. Subsequent radiation oncology follow-ups were at 6 months, 1 year and yearly after for 5 years. Photographs were taken for cosmetic evaluation prior to radiotherapy and during subsequent follow-ups.

\section{Cosmesis assessment}

Cosmetic outcomes were assessed subjectively by the treating physician and the patient. The first cosmetic assessment was done at baseline prior to the start of radiation therapy, then at 6 months and yearly after for 5 years. The overall cosmetic assessment was done using the modified Harvard criteria for cosmesis [15] where a score of 9 or 10 (excellent) was given if the treated breast looks exactly the same as the opposite breast, 7-8 (good) minimal but identifiable effects of radiation; 4-6 (fair) significant effects of radiation on the breast; 1-3 (poor) severe normal tissue sequelae.

Objective cosmesis assessment was performed using BCCT. core software (developed by UNESC at Porto University, Portugal) (Fig. 1). By using the patients' photograph, BCCT. core assesses cosmetic result based on breast symmetry, scar visibility, and skin color changes. The software categories the cosmetic appearance as excellent, good, fair, and poor.

\section{Statistical analysis}

Statistical analyses were performed with the SPSS statistical software package ver. 17.0 (SPSS Inc., Chicago, IL, USA). Descriptive statistics were compiled to characterize the patient populations.

\section{Results}

\section{Patient characteristics and clinical outcome}

Forty-two patients were enrolled in this study from June 2006 to May 2013. One patient withdrew from the study, another patient was determined not to be eligible, and 10 patients did not have photographs at $\geq 2$ years follow-ups. The characteristics of the 30 patients included in the study are shown in Table 1. All patients had negative margins and all of them underwent axillary evaluation. The majority of the patients had estrogen receptor (ER) and progesterone receptor (PR) positive tumors. Use of adjuvant hormonal therapy was left to the discretion of treating medical oncologist. Adjuvant chemotherapy was not used in any of the patients.

The median follow-up time at the time of this analysis was 50.5 months. The 5 -year locoregional control rate was 97.5\%. One patient with BRCA2 mutation had a recurrence in the same quadrant 20 months after radiation. Her disease was initially stage pT1bpNO poorly differentiated invasive ductal carcinoma (IDC), ER/PR positive, and HER2 negative and the recurrence of disease was stage pT1bpNO moderately differentiated IDC, triple negative. At recurrence she was treated with bilateral total mastectomy. The 5-year overall survival rate was 90\%. During the follow-up time period, 4 patients (10\%) died of non-breast cancer related causes without evidence of local recurrence. 
Table 1. Characteristics of 30 enrolled patients

\begin{tabular}{lc}
\hline \multicolumn{1}{c}{ Characteristic } & Value \\
\hline Age (yr) & $72(62-88)$ \\
Histology & \\
IDC & $26(87)$ \\
Other & $4(13)$ \\
Tumor size (cm) & $0.8(0.3-1.7)$ \\
Margin size $(\mathrm{cm})$ & $0.4(0.06-1)$ \\
Number of nodes sampled & $2(1-13)$ \\
T Stage & \\
T1a & $8(27)$ \\
T1b & $14(46)$ \\
T1c & $8(27)$ \\
Grade & \\
G1 & $14(46)$ \\
G2 & $14(46)$ \\
G3 & $2(8)$ \\
ER status & \\
Positive & $28(92)$ \\
Negative & $2(8)$ \\
PR status & \\
Positive & $25(83)$ \\
Negative & $5(17)$ \\
Hormonal therapy & \\
Yes & $23(77)$ \\
No & \\
\hline &
\end{tabular}

Values are presented as mean (range) or number (\%).

IDC, invasive ductal carcinoma; ER, estrogen receptor; PR, progesterone receptor.

Table 2. Dosimetric characteristics of the study population

\begin{tabular}{|c|c|c|c|}
\hline & Mean & Median & Range \\
\hline WBV (mL) & 1461.2 & 1301.5 & $528.5-2551.7$ \\
\hline PTV (mL) & 206.8 & 165.8 & 63.6-828.9 \\
\hline PTV/WBV (\%) & 14.9 & 13.9 & $2.8-38.6$ \\
\hline \multicolumn{4}{|l|}{ WBV (\%) } \\
\hline$V_{5}$ & 73.6 & 74.9 & $30.4-100.0$ \\
\hline$V_{20}$ & 59.3 & 56.5 & $24.0-88.5$ \\
\hline$V_{50}$ & 37.2 & 37.5 & $12.2-71.0$ \\
\hline $\mathrm{V}_{80}$ & 17.7 & 15.0 & $1.1-43.9$ \\
\hline$V_{100}$ & 10.9 & 10.0 & $1.0-25.8$ \\
\hline \multicolumn{4}{|l|}{ WBV-PTV (\%) } \\
\hline$V_{5}$ & 57.2 & 53.0 & $28.1-87.6$ \\
\hline$V_{20}$ & 45.8 & 42.0 & $20.3-74.4$ \\
\hline$V_{50}$ & 28.0 & 28.3 & $11.5-47.9$ \\
\hline$V_{80}$ & 14.1 & 11.0 & $1.2-34.4$ \\
\hline$V_{100}$ & 3.4 & 2.0 & $1.0-8.5$ \\
\hline Dmax (Gy) & 42.7 & 42.6 & $40.4-44.7$ \\
\hline
\end{tabular}

WBV, whole-breast reference volume; PTV, planning target volume; Dmax, maximum dose.

\section{Dosimetric parameters}

Dosimetric radiation treatment parameters are shown in Table 2. The median PTV was $165.8 \mathrm{~mL}$ (range, 63.6 to $828.9 \mathrm{~mL}$ ) and median PTV/WBV was $13.9 \%$. The median $\mathrm{V}_{50}(20 \mathrm{~Gy})$ of the WBV was $37.5 \%$, with the median WBV $V_{100}$ (40 Gy) was $10 \%$ The median $\mathrm{V}_{50}(20 \mathrm{~Gy})$ of the WBV minus PTV was $28.3 \%$ and the median $\mathrm{V}_{100}(40 \mathrm{~Gy}$ ) was $2 \%$. Median Dmax was $42.6 \mathrm{~Gy}$ (range, 40.4 to $44.7 \mathrm{~Gy}$ ).

\section{Cosmetic outcomes}

Overall, during the entire follow-up 89\%-100\% of patients scored their overall cosmetic result as 'excellent' or 'good' (Fig. 2). Similarly, treating physicians rated $89 \%-100 \%$ of patients' cosmesis as 'excellent' or 'good'. The BCCT.core program scored $68 \%-87 \%$ of the of patients' cosmesis as 'excellent' or 'good'. The BCCT.core program scored 'excellent' or 'good' cosmesis at the baseline in $87 \%$ of patients and at the 5 -year followup in $81 \%$ of patients. BCCT.core always scored lower than either patient or physician. The 4\%-6\% of the patients rated their cosmetic outcome as 'poor'. However, no 'poor' outcome was either scored by the BCCT.core program or reported by the physicians. There was not deterioration in the overall cosmetic score after 2 years (Fig. 3).

\section{Discussion and Conclusion}

We previously reported an excellent rate of tumor control and favorable toxicity profiles with daily 2 weeks course of APBR using IMRT [14]. In this study, we have shown the subjective and objective evaluation of cosmesis with dosimetric assessment. APBR is a convenient treatment option in selected patients as it decreases the duration of the treatment. However, because of the lack of randomized clinical trial data, cosmetic outcome with APBR remains controversial. In this prospective trial with a 50.5-month median follow-up, we demonstrated acceptable cosmesis by using both subjective and objective assessment methods. The unique aspects of our study include the objective evaluation of the cosmesis and daily fractionation scheme.

Subjective evaluation by the physicians and/or patient selfassessment is commonly used to report cosmesis. However the results from these methods are not reproducible, influenced by patient's psycho-social adjustment, and not comparable with the cosmesis result of other studies. Objective evaluation of the cosmesis by using a computer program is preferable to eliminate confounders and also compare different study results. To the best to our knowledge, none of the prior studies 


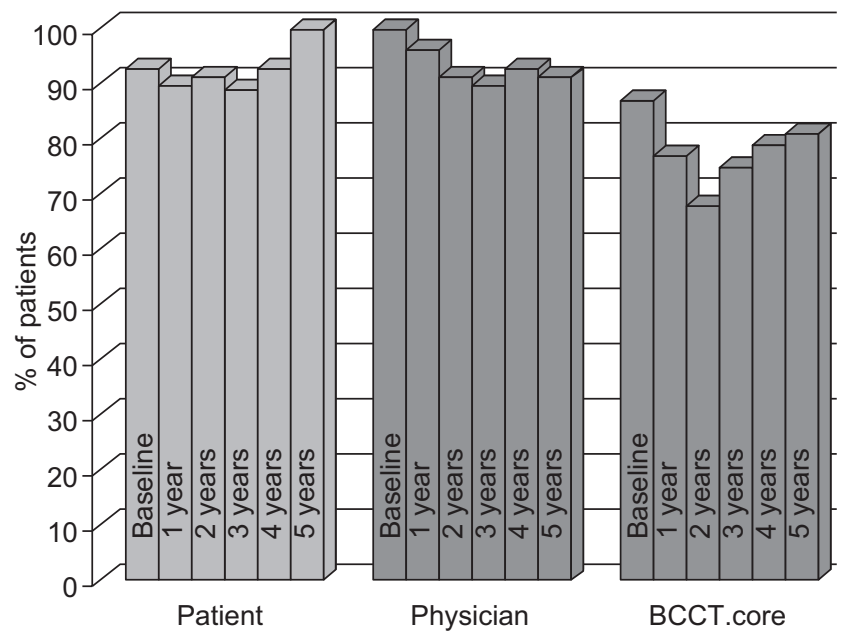

Fig. 2. Patients with excellent or good cosmetic outcomes.

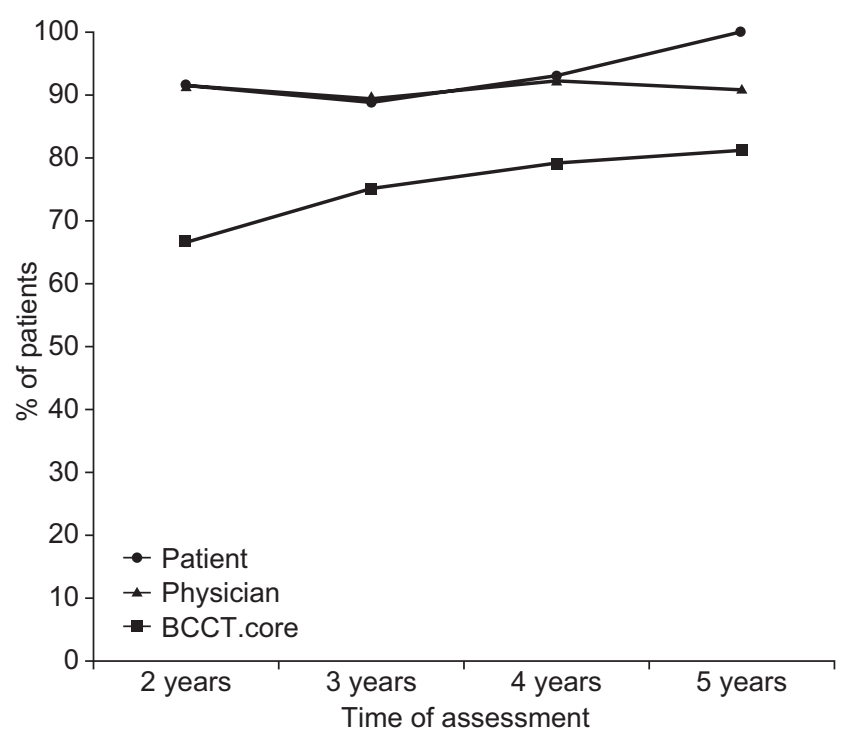

Fig. 3. Patients with excellent or good cosmetic outcomes after 2 years.

Table 3. Comparison with prior APBR with IMRT data

\begin{tabular}{lccc}
\hline \multicolumn{1}{c}{ Parameter } & $\begin{array}{c}\text { Current } \\
\text { study }\end{array}$ & $\begin{array}{c}\text { Univ. of } \\
\text { Miami }\end{array}$ & $\begin{array}{c}\text { Univ. of } \\
\text { Michigan }\end{array}$ \\
\hline Median tumor size $(\mathrm{cm})$ & 0.80 & 0.98 & 0.90 \\
Mean V $_{50} /$ WBV (\%) & 37.2 & 33.4 & 47.9 \\
Mean V100/WBV (\%) & 10.9 & 9.7 & 27.2 \\
Median follow-up (mo) & 50.5 & 44.8 & 60 \\
Excellent or good cosmesis (\%) & & & \\
Patient & 100 & 94 & - \\
Physician & 91 & 97 & 73 \\
BCCT.core & 81 & - & - \\
\hline
\end{tabular}

APBR, accelerated partial breast radiation; IMRT, intensity-modulated radiation therapy; WBV, whole-breast reference volume. with external beam APBR used an objective evaluation method to report cosmesis. Due to the limited studies using IMRT with APBR and no objective cosmetic evaluation, the controversy regarding the acceptable cosmesis remains unresolved. The cosmetic outcomes in our study can be related to the fractionation schedule or dosimetry due to IMRT planning.

Excellent or good cosmesis was reported in 73\%-82\% of patients treated with APBR using 3D-CRT with various fractionation schedule [16-19]. The first study evaluating the outcome of APBR using IMRT with deep inspiration breath hold reported from University of Michigan [4]. Patients in this study received 38.5 Gy in 3.85 Gy fractions given twice daily over 5 consecutive days. Seven out of 34 treated patients (22\%) developed unacceptable cosmesis at a 2-year interim analysis, leading the study to close. Cosmesis was assessed by the physicians. A subsequent reports from the same group reported the continued decline in cosmesis in same cohort at a median follow-up of 5 years [20]. Another study of APBR using IMRT was reported from University of Miami [21]. In this study patient received $38 \mathrm{~Gy}$ in $3.8 \mathrm{~Gy}$ fractions given twice daily over consecutive days. Cosmesis was assessed by both the physicians and the patients. The 'excellent' or 'good' cosmesis was reported by $94 \%$ of the patients and by $97 \%$ of the physicians. These results are similar to the cosmetic outcome in our study.

The important differences between our study and the two previous reports using APBR with IMRT includes the objective assessment of the cosmesis and daily fractionation scheme (Table 3). On the last follow-up, 100\% of the patients scored their overall cosmesis as 'excellent' or 'good' and the physicians rated $91 \%$ of the patients with 'excellent' or 'good' outcomes. BCCT.core program scored $81 \%$ of the patients with 'excellent' or 'good' outcomes. While the objective evaluation with BCCT.core program reports less 'excellent' or 'good' outcomes compared to patients self-assessment and physicians rating, the baseline cosmesis assessment with BCCT. core is similar with assessment at the last follow up, indicating that the cosmesis was not compromised with the treatment. Furthermore, unlike the reports from Liss et al. [20] and Lei et al. [22] there is no trend toward continued decline in cosmesis over time.

In this current study, patients received $40 \mathrm{~Gy}$ in $4 \mathrm{~Gy}$ daily fractions. The daily fractionation schedule logistically is easier for patients particularly elderly and if there is long distance travel involved. Similar to prior reports, we used the $\alpha / \beta$ value of $4 \mathrm{~Gy}$ to determine the tumor control biological equivalent dose (BED) and the $\alpha / \beta$ value of 2 and $10 \mathrm{~Gy}$ for 
the toxicities [23]. The tumor control BED value in our study was $80 \mathrm{~Gy}$, which was in the range of BED values (72-99 Gy) from different standard WBR protocols. Calculated BED values in our study were lower than the standard WBR protocols for $\alpha / \beta$ of 10 and slightly higher for $\alpha / \beta$ of 2 . Unlike prior studies using bid schedule, use of daily fractionation in our study may allow adequate time for breast tissue to recover, which may further explain the better cosmesis in our study. The mean $V_{5}(2$ Gy) of the WBV was $73.6 \%$ in our study and this is similar to prior APBR with 3D-CRT studies (74\%-79\%) [19,24]. The mean $V_{20}$ (8 Gy) of the WBV was $59.3 \%$ in our study and this is again similar to prior APBR with 3D-CRT studies (58\%-66\%) [19,24]. The mean $\mathrm{V}_{50} /$ WBV and V100/WBV in our study is lower than the report from University of Michigan (37.2\% vs. $47.9 \%$ and $10.9 \%$ vs. $27.2 \%$, respectively) as well as the median PTV $\left(165.8 \mathrm{mc}^{3}\right.$ vs. $\left.185.8 \mathrm{mc}^{3}\right)$. On the basis of these difference, we hypothesize that cosmesis may be dependent in part on PTV size, $V_{50} / W B V$ and $V_{100} / W B V$, and use of daily fractionation.

In conclusion, APBR is an evolving standard of care and the debate over the assessment methods of the cosmetic outcome will likely remain unresolved until large randomized clinical trials are designed to compare them. Nevertheless, a favorable cosmesis in our study supports the use of APBR with IMRT with a daily 2-week schedule as a promising treatment option for elderly patients with early stage breast cancer.

\section{Conflict of Interest}

No potential conflict of interest relevant to this article was reported.

\section{References}

1. Arthur DW, Winter K, Kuske RR, et al. A Phase II trial of brachytherapy alone after lumpectomy for select breast cancer: tumor control and survival outcomes of RTOG 95-17. Int J Radiat Oncol Biol Phys 2008;72:467-73.

2. Cuttino LW, Keisch $M$, Jenrette JM, et al. Multi-institutional experience using the MammoSite radiation therapy system in the treatment of early-stage breast cancer: 2-year results. Int J Radiat Oncol Biol Phys 2008;71:107-14.

3. Vicini F, Winter K, Wong J, et al. Initial efficacy results of RTOG 0319: three-dimensional conformal radiation therapy (3D-CRT) confined to the region of the lumpectomy cavity for stage I/ II breast carcinoma. Int J Radiat Oncol Biol Phys 2010;77:11207.

4. Jagsi R, Ben-David MA, Moran JM, et al. Unacceptable cosmesis in a protocol investigating intensity-modulated radiotherapy with active breathing control for accelerated partial-breast irradiation. Int J Radiat Oncol Biol Phys 2010;76:71-8.

5. Oliver $\mathrm{M}$, Chen J, Wong E, Van Dyk J, Perera F. A treatment planning study comparing whole breast radiation therapy against conformal, IMRT and tomotherapy for accelerated partial breast irradiation. Radiother Oncol 2007;82:317-23.

6. Rusthoven KE, Carter DL, Howell K, et al. Accelerated partialbreast intensity-modulated radiotherapy results in improved dose distribution when compared with three-dimensional treatment-planning techniques. Int J Radiat Oncol Biol Phys 2008;70:296-302.

7. Saibishkumar EP, Mackenzie MA, Severin D, et al. Skin-sparing radiation using intensity-modulated radiotherapy after conservative surgery in early-stage breast cancer: a planning study. Int J Radiat Oncol Biol Phys 2008;70:485-91.

8. Al-Ghazal SK, Blamey RW. Cosmetic assessment of breastconserving surgery for primary breast cancer. Breast 1999;8:162-8.

9. Pezner RD, Lipsett JA, Vora NL, Desai KR. Limited usefulness of observer-based cosmesis scales employed to evaluate patients treated conservatively for breast cancer. Int J Radiat Oncol Biol Phys 1985;11:1117-9.

10. Al-Ghazal SK, Fallowfield L, Blamey RW. Does cosmetic outcome from treatment of primary breast cancer influence psychosocial morbidity? Eur J Surg Oncol 1999;25:571-3.

11. Heil J, Dahlkamp J, Golatta $M$, et al. Aesthetics in breast conserving therapy: do objectively measured results match patients' evaluations? Ann Surg Oncol 2011;18:134-8.

12. Cardoso JS, Cardoso MJ. Towards an intelligent medical system for the aesthetic evaluation of breast cancer conservative treatment. Artif Intell Med 2007;40:115-26.

13. Cardoso MJ, Cardoso JS, Oliveira HP, Gouveia P. The breast cancer conservative treatment. Cosmetic results - BCCT.core - Software for objective assessment of esthetic outcome in breast cancer conservative treatment: a narrative review. Comput Methods Programs Biomed 2016;126:154-9.

14. Sayan M, Wilson K, Nelson C, Gagne H, Rubin D, Heimann R. A novel schedule of accelerated partial breast radiation using intensity-modulated radiation therapy in elderly patients: survival and toxicity analysis of a prospective clinical trial. Radiat Oncol J 2017;35:32-8.

15. Rose MA, Olivotto I, Cady B, et al. Conservative surgery and radiation therapy for early breast cancer: long-term cosmetic results. Arch Surg 1989;124:153-7.

16. Shah C, Wilkinson JB, Lanni T, et al. Five-year outcomes 
and toxicities using 3-dimensional conformal external beam radiation therapy to deliver accelerated partial breast irradiation. Clin Breast Cancer 2013;13:206-11.

17. Berrang TS, Olivotto I, Kim DH, et al. Three-year outcomes of a Canadian multicenter study of accelerated partial breast irradiation using conformal radiation therapy. Int J Radiat Oncol Biol Phys 2011;81:1220-7.

18. Olivotto IA, Whelan TJ, Parpia $S$, et al. Interim cosmetic and toxicity results from RAPID: a randomized trial of accelerated partial breast irradiation using three-dimensional conformal external beam radiation therapy. J Clin Oncol 2013;31:403845.

19. Leonard KL, Hepel JT, Hiatt JR, Dipetrillo TA, Price LL, Wazer $D E$. The effect of dose-volume parameters and interfraction interval on cosmetic outcome and toxicity after 3-dimensional conformal accelerated partial breast irradiation. Int J Radiat Oncol Biol Phys 2013;85:623-9.

20. Liss AL, Ben-David MA, Jagsi $R$, et al. Decline of cosmetic outcomes following accelerated partial breast irradiation using intensity modulated radiation therapy: results of a single-institution prospective clinical trial. Int J Radiat Oncol Biol Phys 2014;89:96-102.

21. Lewin $A A$, Derhagopian $R$, Saigal $K$, et al. Accelerated partial breast irradiation is safe and effective using intensitymodulated radiation therapy in selected early-stage breast cancer. Int J Radiat Oncol Biol Phys 2012;82:2104-10.

22. Lei RY, Leonard $C E$, Howell $K T$, et al. Four-year clinical update from a prospective trial of accelerated partial breast intensitymodulated radiotherapy (APBIMRT). Breast Cancer Res Treat 2013;140:119-33.

23. Williams MV, Denekamp J, Fowler JF. A review of alphal beta ratios for experimental tumors: implications for clinical studies of altered fractionation. Int J Radiat Oncol Biol Phys 1985;11:87-96.

24. Shaitelman SF, Kim LH, Grills IS, et al. Predictors of long-term toxicity using three-dimensional conformal external beam radiotherapy to deliver accelerated partial breast irradiation. Int J Radiat Oncol Biol Phys 2011;81:788-94. 